\title{
El aprendizaje mediante BreakoutEdu en ciencias jurídicas: humor y juego en el aula. Una experiencia en la Universitat Politècnica de València
}

\author{
Francisca Ramón Fernández ${ }^{\mathrm{a}}$, Cristina Lull Noguera ${ }^{\mathrm{b}}$, Desamparados Soriano Soto ${ }^{\mathrm{c}}$, Laura García- \\ España Soriano $^{d}$ y Laura Osete Cortina ${ }^{e}$ \\ aProfesora titular. Departamento de Urbanismo, Universitat Politècnica de València, frarafer@urb.upv.es, ${ }^{b}$ Profesora \\ contratada doctora, Departamento de Química, Universitat Politècnica de València, clull@upvnet.upv.es, ${ }^{\mathrm{c} C a t e d r a ́ t i c a . ~}$ \\ Departamento de Producción Vegetal, Universitat Politècnica de València, asoriano@prv.upv.es, ${ }^{\mathrm{d}}$ Profesora Instituto. \\ Universitat de València, laugars2@alumni.uv.es, ${ }^{e}$ Profesora asociada, Departamento de Conservación y Restauración \\ de Bienes Culturales, Universitat Politècnica de València, losete@crbc@upv.es
}

\begin{abstract}
The innovation and results obtained from designing and applying a BreakoutEdu based on the regulations of Valencian Civil Law are presented, to promote legal concepts learning related to the Valencian Huerta. Through three fictitious families that develop their activity in the agricultural field, an interactive game is designed for the students of Civil Law II of the Master's Degree in Administrative Management. The results observed included: an improvement in learning and a greater revitalization in the classroom, in addition to favouring the concern for the applicable legislation. We consider that the attractive comic-type design of the structure of the activity helps to capture the attention of students and improves their cognitive ability. The experience can be extrapolated to other subjects, since the design focuses on reusable, non-contextualized concepts.
\end{abstract}

Keywords: Humor, educational gamification; breakout, civil law, Valencian orchard, water, learning, skills, innovation

\section{Resumen}

Se presenta la innovación y resultados obtenidos de diseñar y aplicar un BreakoutEdu basado en la normativa de Derecho civil valenciano, para favorecer el aprendizaje de conceptos jurídicos relacionados con la Huerta valenciana. A través de tres familias ficticias que desarrollan su actividad en el ámbito agrario, se diseña un juego interactivo para los alumnos de Derecho civil II del Máster en Gestión Administrativa. Los resultados observados son una mejora en el aprendizaje y una mayor dinamización en el aula, además de favorecer la inquietud por la legislación aplicable. Consideramos que el diseño atractivo tipo cómic de la estructura de la actividad, ayuda a captar la atención del alumnado y mejora su capacidad cognitiva. La experiencia se puede extrapolar a otras asignaturas, ya que el diseño se centra en conceptos reutilizables, y no contextualizados.

Palabras clave: Humor, gamificación educativa, breakout, derecho civil, Huerta de València, aguas, aprendizaje, competencias, innovación

\section{Introducción}

Dentro del desarrollo del PIME que hemos llevado a cabo durante los cursos académicos 2018-2020, referente al "Diseño de materiales docentes basados en recursos audiovisuales de humor para la mejora del aprendizaje y su evaluación en ciencias sociales y jurídicas", nos hemos planteado el diseño de distintos 
materiales para la innovación docente. Siguiendo en la línea de innovación docente del grupo RETAJUDOCA (Recursos tecnológicos para el aprendizaje jurídico, la documentación y la comunicación audiovisual) de la Universitat Politècnica de València en la que desarrollamos materiales para la mejora docente en ciencias sociales y jurídicas a través de la utilización de elementos visuales (Cabedo et al., 2016, 2017, 2018 y 2019), hemos considerado que la gamificación podría resultar atractiva al alumnado de las titulaciones en las que se imparte asignaturas con un perfil jurídico. En estos contextos educativos, además, se ha observado una baja participación y motivación del alumnado, así como la dificultad en el aprendizaje de estos conceptos, asociada a que los alumnos carecen de una formación jurídica sólida.

En este sentido, la gamificación en el aula resulta especialmente atractiva al alumnado, ya que el juego siempre facilita el aprendizaje y convierte la dinámica habitual en una oportunidad para poder incrementar la participación del alumnado, así como su motivación en el aula (Prieto, 2020). En el ámbito de las ciencias sociales y jurídicas no ha sido muy habitual la introducción de elementos lúdicos en la denominada enseñanza tradicional, sin embargo, en los últimos años sí que se ha producido una apertura a los recursos informáticos que utilizan el juego como estrategia de aprendizaje. Es el caso de programas como FlipQuiz, Socrative, uLearn-Play o Kahoot! (Ramón et al., 2020).

Un BreakoutEdu de manera sencilla es un juego en el que los jugadores trabajan en equipo resolviendo actividades/pruebas/acertijos con diferentes pistas que los llevarán a abrir una caja con candado en la que se encuentra la pista final (Rouse, 2017). De acuerdo con Negre (2017) el BreakoutEdu es una microgamificación que fomenta el aprendizaje significativo y que tiene multiples ventajas como desarrollar el pensamiento crítico y la habilidad para resolver problemas, promocionar el trabajo en equipo, construir pensamiento deductivo, etc. Hoy en día, hay docentes universitarios que gamifican en sus aulas con BreakoutEdu con experiencias muy positivas (Bernabeu, 2018; Detwiler et al., 2018; O’Brien y Pitera, 2019; Queiruga-Dios et al., 2020).

En el caso de las asignaturas jurídicas supone un gran reto no sólo por el diseño de la actividad, sino también por el número de alumnado que suele ser numeroso (Ramón et al., 2020). No obstante, teniendo en cuenta dichas premisas, estuvimos motivados para diseñar nuestro propio juego, adaptándolo a las características de la asignatura donde lo íbamos a aplicar. En este caso, teniendo en cuenta la legislación de Derecho civil valenciano, se ha diseñado un BreakoutEdu basado en tres familias, con un diseño tipo cómic, para atraer la atención del alumnado, y con la finalidad de potenciar un autoaprendizaje dinámico en el aula. Esta experiencia educativa constituye una primera aproximación en relación con la implantación de dinámicas basadas en el juego, para obtener información que nos indique el grado de aceptación del alumnado y los beneficios que aporta, con el fin de implementarla en otros grupos y contextos más amplios.

Se expondrán los resultados obtenidos en la experiencia que hemos desarrollado en el presente curso académico 2019-2020, tras diseñar y utilizar un BreakoutEdu relativo a "Las familias en la Huerta" en la titulación del Máster en Gestión Administrativa, en concreto en la asignatura "Derecho civil II" con la finalidad de observar el grado de aprendizaje y su mejora por parte de los alumnos, tras su realización y contestación a una encuesta de preguntas cerradas.

\section{Objetivos}

Dentro del proyecto que nos ocupa se ha construido un BreakoutEdu con el objetivo de reforzar los conocimientos adquiridos en la asignatura, ponerlos en práctica y realizarlo de forma atractiva para los alumnos.

Para ello se ha preparado un BreakoutEdu educativo para alumnos universitarios. Breakout significa fugarse, salir de algo, aplicado a la enseñanza significaría resolver, y es ampliamente utilizado en la innovación docente con la finalidad de gamificar. 
Son numerosas las aplicaciones educativas que existen en la actualidad, y dentro de ellas el BreakoutEdu se ha integrado con fuerza en el medio educativo.

Los principales objetivos son los siguientes:

a) Utilizar un BreakoutEdu diseñado de forma atractiva, con inclusion de dibujos con notas de humor sobre una legislación actual, teniendo como base el empleo de recursos visuales en la línea de los PIMEs que desarrolla el grupo.

b) Incrementar la participación y dinamización en el aula del alumnado de titulación no jurídica, pero que debe aprender conceptos jurídicos.

c) Validar la experiencia de innovación docente como apta y comprobar a través de los resultados de una encuesta que se proporciona después de la actividad si el alumnado tiene una recepción positiva a la actividad y mejora la comprensión e integración de conceptos.

d) Observar la actividad grupal en el aula, y la capacidad del alumnado de involucrarse en una actividad gamificada dentro de la asignatura.

\section{Diseño de la innovación: ¿Cómo hemos elaborado el BreakoutEdu de las familias de la Huerta?}

Para esta innovación que presentamos hemos realizado un diseño que resultara muy atractivo para el juego del BreakoutEdu. En este sentido, se han diseñado varios recursos como ha sido una sopa de letras y unos dibujos tipo cómic de humor para representar varias familias de la Huerta, que teniendo en cuenta sus características e indumentaria el alumnado pudiera relacionarlas con diversos conceptos jurídicos que teníamos interés en el aprendizaje.

La innovación que se ha realizado ha sido diseñada a partir de una sopa de letras, mediante la que se inicia el BreakoutEdu (Fig. 1), y que hemos ajustado a la asignatura en la que lo queríamos aplicar, incorporando además, diversos elementos visuales y audiovisuales de humor.

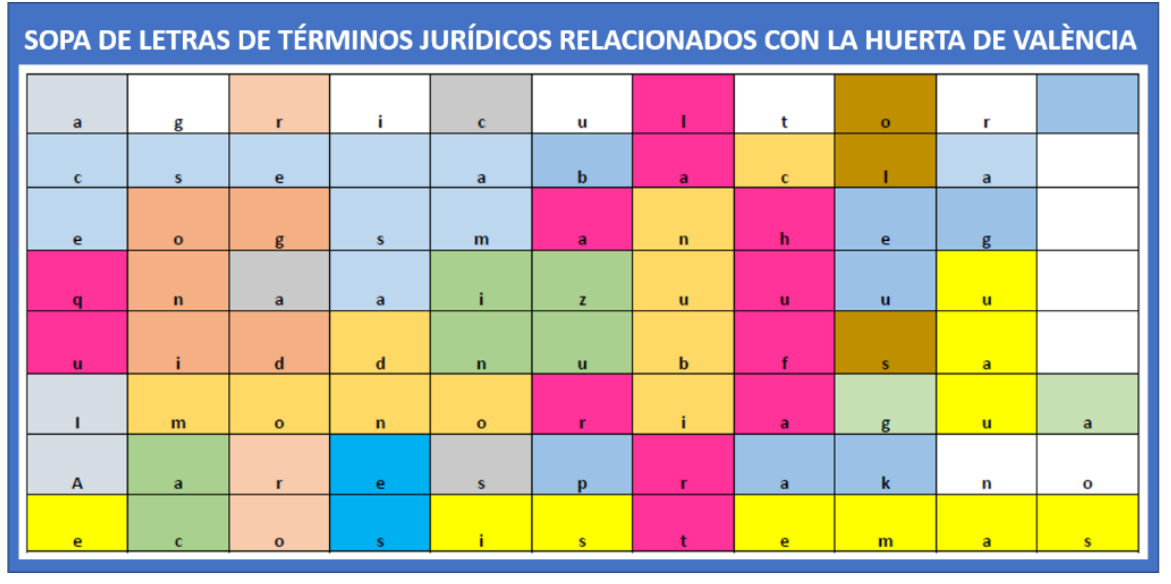

Fig. 1. Sopa de letras diseñada para el breakout educativo. Fuente: Elaboración propia

\subsection{Elementos visuales a través de una serie de dibujos tipo cómic}

Para diseñar la actividad se elaboraron tres dibujos que representaban a tres familias, y se eligió que fueran tipo cómic:

a) Familia Santahuerta (Figura 2), tiene como característica que está integrada por un solo miembro, y que se representa junto a su animal de compañía, un perro. Se trata, por su indumentaria, de un labrador 
El aprendizaje mediante BreakoutEdu en ciencias jurídicas: humor y juego en el aula. Una experiencia en la Universitat Politècnica de València

valenciano, que se dedica al cultivo de productos destinados al comercio de proximidad. Tiene un huerto no demasiado extenso, y probablemente sea un arrendamiento histórico valenciano.

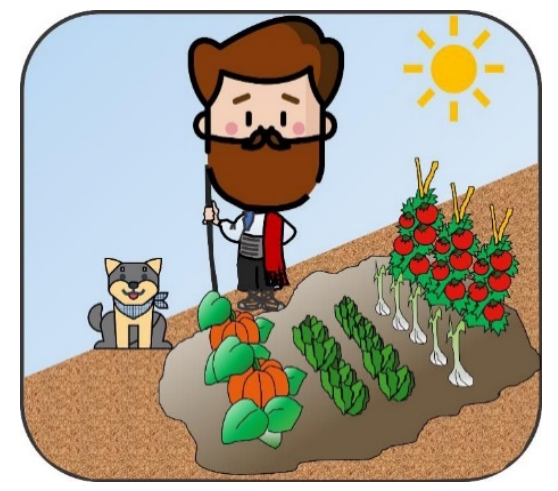

Fig. 2. Familia Santahuerta. Fuente: Laura Osete Cortina

b) Familia Santaermita (Figura 3), está integrada por tres miembros, padre, madre y descendiente. Por su indumentaria tienen una clase social elevada, posiblemente sean arrendadores, y posean bienes protegidos en la Huerta, como Bien de interés cultural o Bienes de relevancia local.

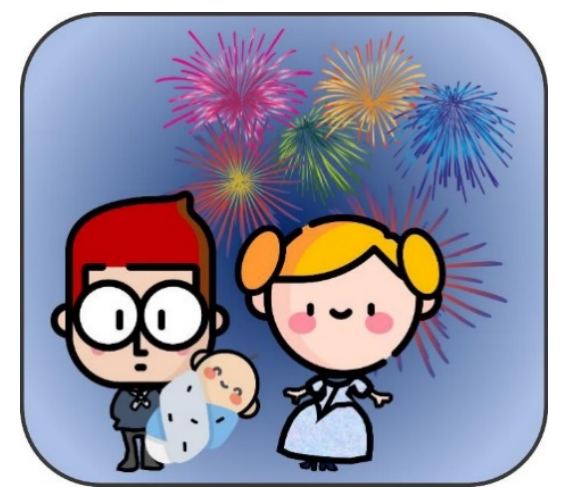

Fig. 3. Familia Santaermita. Fuente: Laura Osete Cortina

c) Familia Santaacequia (Figura 4), se compone de una pareja de avanzada edad, que por su indumentaria no es de alto nivel económico, y que viven en una edificación propia de la Huerta, como es la barraca. Disponen de tierras, y las cultivan ellos mismos, con las obligaciones propias de los agricultores. Las tierras son de su propiedad, y tienen un gran apego al campo, ya que han vivido toda su vida en dicho ámbito.

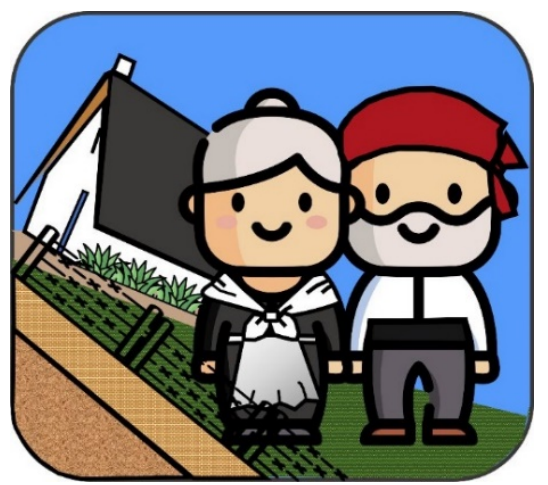

Fig. 4. Familia Santaacequia. Fuente: Laura Osete Cortina 
Estas tres familias constituyen los elementos clave para personalizar y contextualizar casos prácticos que implican contratos agrarios, arrendamientos históricos o elementos de patrimonio cultural relacionados con la Huerta de València.

\subsection{Elementos académicos y conceptos de aprendizaje incluidos}

Se diseñaron unas cajas de Breakout, para que los alumnos a través del itinerario propuesto en clase resolvieran el reto que les llevaría a la apertura de la caja donde se encontraba. Los alumnos parten de un conocimiento previo de los temas impartidos en clase y a través del BreakoutEdu se quiere incidir en mayor manera ya sea por su interés, por su importancia o porque son atractivos para el alumno y queremos reforzar sus conocimientos.

Para ello se prepararon unas preguntas que se montaron en una hoja Excel autocorrectiva (Figura 5), de manera que al responderla el programa indique si la respuesta es o no correcta. Es decir, se trata de una actividad autocorrectiva, en la que el alumnado teniendo en cuenta los conceptos previamente explicados, y pudiendo consultar los materiales de la asignatura, las respondía en una hoja y automáticamente sabía si la respuesta era o no correcta.

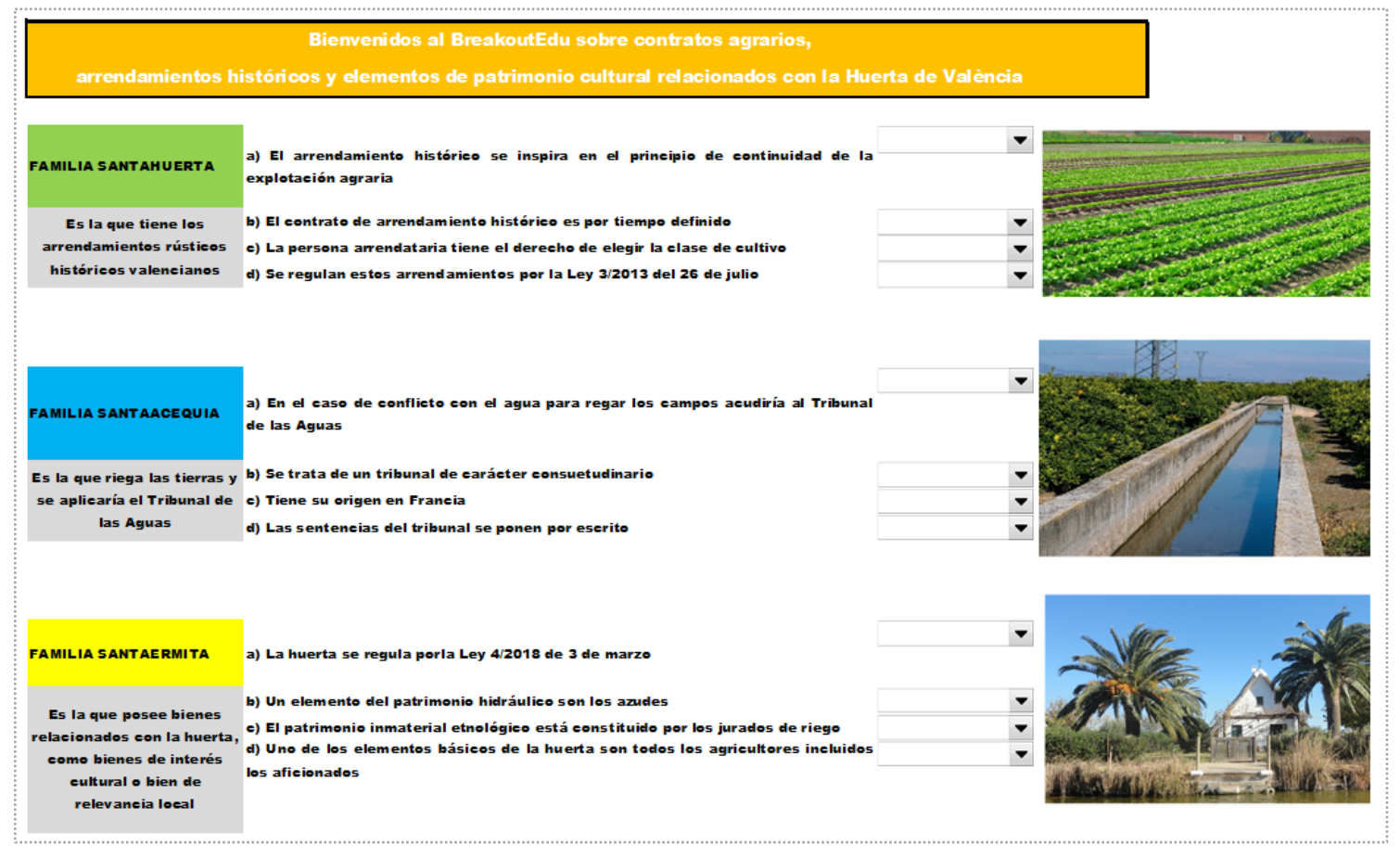

Fig. 5. Diseño del excel conteniendo las cuestiones autocorrectivas. Fuente: elaboración propia. Imágenes: Pixabay (1 y 2) y Francisca Ramón Fernández (3)

El alumno leerá las preguntas para poder superar el reto. Para ayudar a resolverlas se le darán pistas que encontrará en un video de una página web, en un cómic, en los apuntes o en la bibliografía de la asignatura. Cuando consiga responder a las primeras preguntas de menor dificultad el programa le facilitará una clave. Dicha clave abrirá el candado de unas cajas preparadas en cuyo interior en sobres escritos con tinta mágica (se lee solo al aplicar luz ultravioleta con unos bolígrafos que se le facilitará a cada grupo) podrá obtener más pistas que le ayudaran a la resolución del resto de preguntas.

Metodológicamente se pueden poner una o varias cajas con sus correspondientes candados donde encontrará mayor número de pistas en función de la dificultad de la prueba. Se utilizaron candados 
numéricos que se configuraron para la prueba. De esta forma, hemos conseguido con este sencillo diseño que el alumnado viera la actividad como muy interesante y atractiva, ya que se trataba de superar retos para pasar a la siguiente prueba, y los elementos como las cajas y los candados favorecían una implicación del alumnado mucho más intensa que si la actividad la hubiéramos realizado con otros elementos en el juego.

De esta forma, con todo este material, en clase se creó un BreakoutEdu propio. En este caso las pruebas se centraron en La Ley 3/2013, de 26 de julio, de los Contratos y otras relaciones jurídicas agrarias (BOE núm. 222, de 16 de septiembre de 2013), modificada por Ley 2/2019, de 6 de febrero, de la Generalitat, de reforma de la Ley $3 / 2013$, de 26 de julio, de los contratos y otras relaciones jurídicas agrarias, para exigencia de la forma escrita y para la creación del registro de operadores, contratos y relaciones jurídicas agrarias (BOE núm. 51, de 28 de febrero de 2019), y la Ley 5/2018, de 6 de marzo, de la Huerta de València (BOE núm. 96, de 20 de abril de 2018), haciendo hincapié principalmente en las figuras jurídicas reguladas como son los contratos agrarios, los arrendamientos históricos, los elementos de patrimonio cultural relacionados con la huerta de Valencia y el Decreto 219/2018, de 30 de noviembre, del Consell, por el que se aprueba el Plan de acción territorial de ordenación y dinamización de la Huerta de València (DOGV núm. 8448, de 20 de diciembre de 2018).

\section{Desarrollo de la innovación y su aplicación en la asignatura Derecho civil II, en el Máster en Gestión Administrativa de la Universitat Politècnica de València}

La utilización de la actividad tuvo como pretensión la mejora del aprendizaje de conceptos jurídicos muy concretos y determinados, teniendo en cuenta el perfil del alumnado del Máster. También se relacionó con el Objetivo de Desarrollo Sostenible (ODS), número 16: "Paz, justicia e instituciones sólidas", ya que en el programa de la asignatura se estudian las principales instituciones del Derecho civil valenciano relacionadas con la justicia.

La innovación se aplicó al segundo cuatrimestre del Máster en Gestión Administrativa, que se imparte en la Facultad de Administración y Dirección de Empresas de la Universitat Politècnica de València. Es una asignatura obligatoria del primer curso, y durante el curso 2019-2020 tiene un total de 16 alumnos.

Las competencias de la asignatura orientadas a la futura actividad profesional son las siguientes:

CB6(GE) Poseer y comprender conocimientos que aporten una base u oportunidad de ser originales en el desarrollo y/o aplicación de ideas, a menudo en un contexto de investigación.

CE9(GE) Capacidad para asesorar y tramitar profesionalmente contrato de terceros privados o susceptibles de representación ante las administraciones públicas.

CB9(GE) Desarrollar las destrezas que permitan comunicar sus conclusiones $-\mathrm{y}$ los conocimientos y razones últimas que las sustentan- a públicos especializados y no especializados de un modo claro y sin ambigüedades

La competencia transversal que se trabaja y que es punto de control en esta asignatura es la (CT1) "Comprensión e integración" con la que se pretende que el alumnado demuestre una comprensión e integración del conocimiento dentro de la contextualización de la propia asignatura, como también más allá de la misma. Esta competencia en el caso del Máster de Gestión Administrativa resulta fundamental, así como la asignatura punto de control de la misma, Derecho civil II, permite identificar y diferenciar los conceptos más básicos del ámbito jurídico especializado en material de contratación y relaciones jurídicas agrarias. La utilización de recursos visuales y audiovisuales en el aprendizaje resulta una herramienta 
fundamental para el diseño de los casos, ya que a través de la historia reflejada y creada en el BreakoutEdu el alumnado va a ser capaz de relacionar conceptos jurídicos insertándolos en un entorno real y práctico.

La realización de la actividad se lleva a cabo en el aula, durante una sesión de aproximadamente una hora, durante la asignatura de Derecho civil II, con un total de 16 alumnos.

\section{Resultados obtenidos}

Se realizó la actividad en la asignatura Derecho civil II, de la titulación del Máster en Gestión Administrativa que se imparte en la Facultad de Administración y Dirección de Empresas, de la Universitat Politècnica de València.

Los alumnos que participaron fueron 5 de un total de 16 matriculados, y estuvieron muy participativos e interesados por la actividad.

Después de la realización de la actividad, se les pasó una encuesta para obtener datos sobre la percepción de la actividad, así como sus impresiones de la misma.

Se les formularon las siguientes cuestiones, cerradas, a las que debían contestar en una escala del 1 al 5 , siendo 1 la más baja puntuación y 5 la máxima puntuación, teniendo en cuenta las características de las preguntas formuladas:

1. Interés de la actividad

2. Dificultad de la actividad

3. Aprendizaje de conceptos con la actividad

4. Diversión de la actividad

5. Conocimiento anterior de este tipo de actividades

6. Realización de más actividades de este tipo

7. Recomendación de esta actividad

Los resultados obtenidos los hemos indicado en las Figuras 6, 7 y 8 :

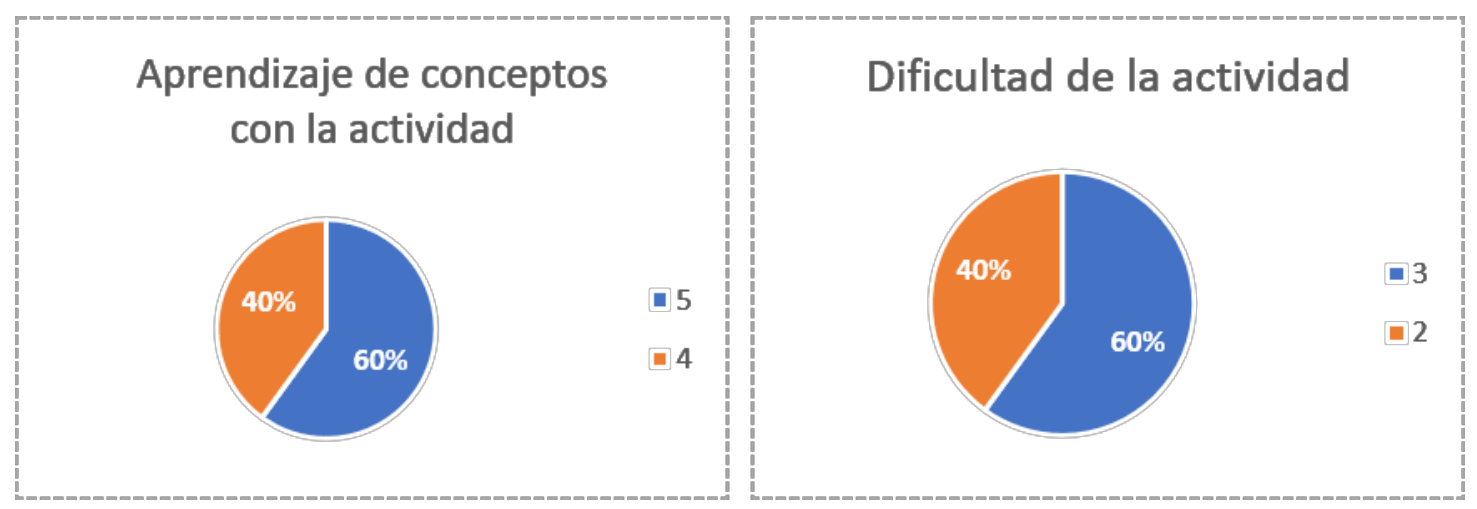

Fig. 6. Resultado encuesta realizada tras la actividad. $N=5$. El alumno calificó del 1 al 5 , siendo el 1 y el 5 las puntuaciones más baja y alta respectivamente, en una escala de 5 puntos. 

en la Universitat Politècnica de València

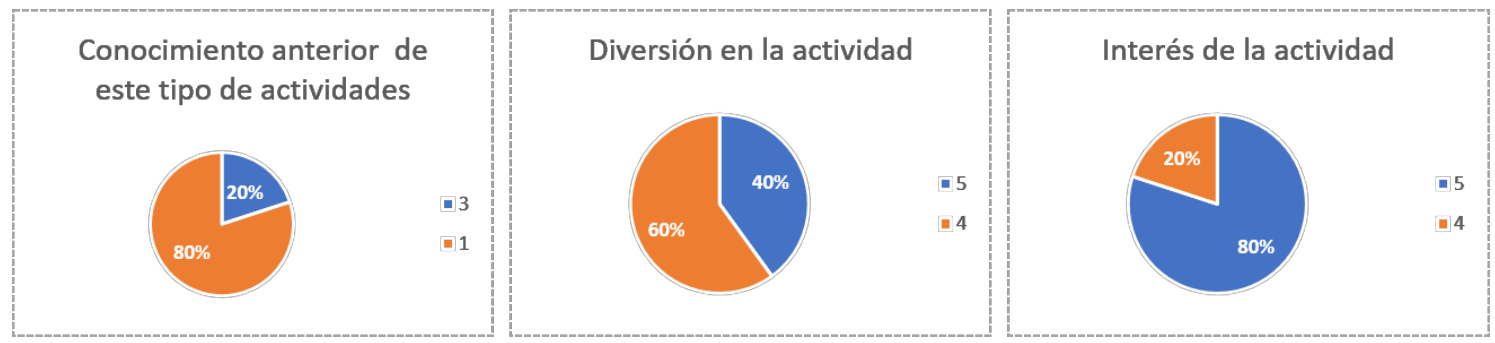

Fig. 7. Resultado encuesta realizada tras la actividad. $N=5$. El alumno calificó del 1 al 5 , siendo eI 1 y el 5 las puntuaciones más baja y alta respectivamente, en una escala de 5 puntos.
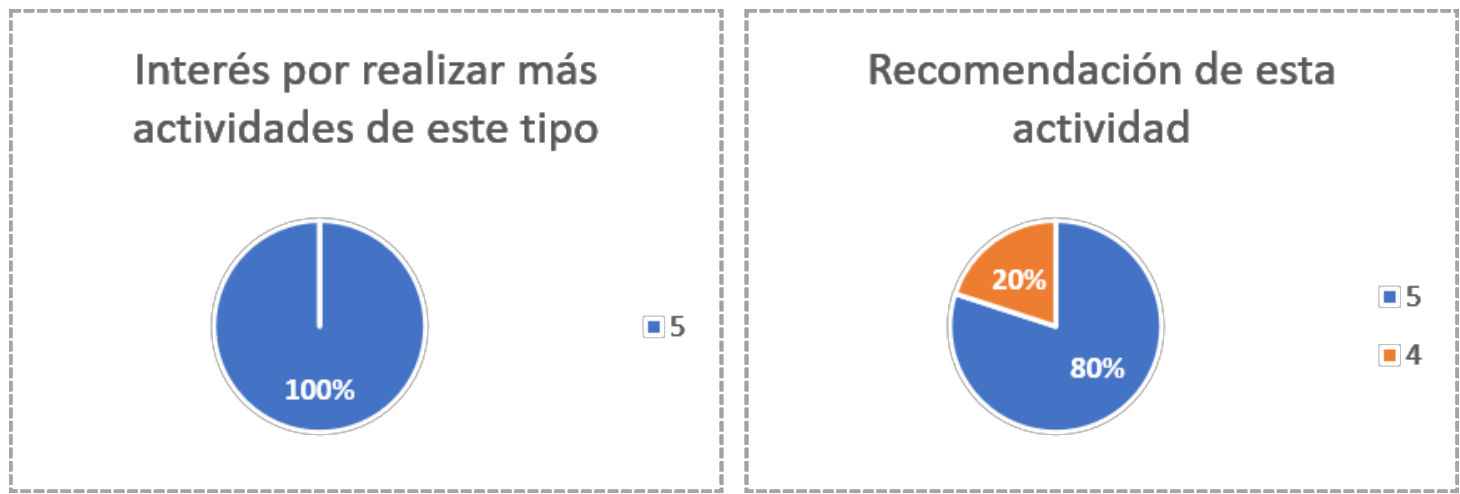

Fig. 8. Resultado encuesta realizada tras la actividad. $N=5$. El alumno calificó del 1 al 5 , siendo el 1 y el 5 las puntuaciones más baja y alta respectivamente, en una escala de 5 puntos.

Por último, en la encuesta se realizó una pregunta de respuesta abierta referente a los comentarios que

quisieran realizar libremente el alumnado sobre la actividad, y las respuestas fueron las siguientes:

-"Es una actividad que permite reflexionar sobre conocimientos básicos y ampliarlos de una forma divertida, aprendizaje mediante la diversión y recordar conceptos gracias a esta experiencia".

-"Me parece una forma interesante de aprender conceptos. Entretenido, una forma distinta de aprender"

-"Interesante-entretenido-modo de aprendizaje efectivo-recomendación de esta actividad"

-"Los juegos siempre motivan y a la hora de poner en prácticas conocimientos adquiridos es muy útil para captar nuestra atención y divertirnos aprendiendo".

Si bien los resultados que hemos obtenido en esta experiencia son de un número reducido de alumnos, demuestran la amplia aceptación del alumnado y nos alientan a implementar dicha innovación en los sucesivos cursos académicos.

También consideramos que la dinámica propuesta es extrapolable a otras asignaturas de grado y no solamente de máster, adaptándola teniendo en cuenta las limitaciones de las aulas para poder llevar a cabo la misma.

\section{Conclusiones}

La utilización del BreakoutEdu en la docencia incrementa la participación del alumnado en el aula, y es una de las mejores herramientas dentro de la gamificación para la dinamización de una asignatura. Supone una fórmula idónea para motivar al alumnado, ya que se involucra en un juego que incluye un componente lúdico que no es habitual en las actividades que se realizan en el aula (Corchuelo, 2018; Moreno et. al., 2019).

En el caso que hemos aplicado el BreakoutEdu referente a las familias en la huerta hay que indicar que el diseño en sí ya ha supuesto un esfuerzo por parte del profesorado de adaptación a una actividad que no es habitual utilizarla en el ámbito de la docencia jurídica. Sin embargo, la gamificación se hace cada vez más 
visible en el aula, con independencia de la materia objeto de estudio (García, 2018; Liarte, 2018; Moreno, 2019; Manzano-León et al., 2020).

La experiencia realizada puede ser completada con la utilización de los audiovisuales como herramienta de aprendizaje, tal y como hemos empleado con mayor frecuencia, ya que son elementos o recursos perfectamente compatibles entre sí. E incluso se puede mostrar al alumnado un audiovisual previo para resolver un BreakoutEdu a semejanza de un caso práctico (Oltra et al., 2016; Pérez et al., 2017; Ramón et al., 2016a, 2016b, 2019a y 2019b). Consideramos que dicha combinación podría incrementar la motivación del alumnado, y mejorar su aprendizaje, además de poder ser utilizada la actividad del BreakoutEdu para la evaluación de las competencias transversales.

Los resultados obtenidos en la actividad realizada en el BreakoutEdu han sido altamente positivos, y los alumnos han tenido una percepción de la actividad de incremento de la forma de aprendizaje, más divertida y amena, ya que cualquier actividad de gamificación supone un aliciente en el estudio de los conceptos. También resaltan la capacidad de recuerdo de los conceptos facilitados a través de la actividad.

La experiencia mostrada en este estudio constituye una prueba previa, que, dado el positivo resultado obtenido y la aceptación por parte del alumnado, se va a aplicar a un mayor número de muestras en cursos posteriores.

\section{Agradecimientos}

Trabajo realizado en el marco del Proyecto de Innovación y Mejora Educativa (PIMEs) "Diseño de materiales docentes basados en recursos audiovisuales de humor para la mejora del aprendizaje y su evaluación en ciencias sociales y jurídicas", presentado en el marco de la convocatoria de Proyectos de Innovación y Mejora Educativa (PIMEs) llevada a cabo en la Universitat Politècnica de València para el curso 2017-2018 obteniendo resolución favorable de la Comisión de Evaluación y Seguimiento de Proyectos de Innovación y Convergencia (CESPIC) en su sesión de 24 de julio de 2018 y concedido por el Vicerrectorado de Estudios y Convergencia Europea de la Universitat Politècnica de València. Años 20182020. Investigadora principal: Francisca Ramón Fernández. Investigadores: Vicente Cabedo Mallol, María Emilia Casar Furió, Vicent Giménez Chornet, Cristina Lull Noguera y Juan Vicente Oltra Gutiérrez, Enrique Orduña Malea y Amparo Soriano Soto y en el marco del Proyecto I+D+i RTI2018-097354-B-100. (2019-2022), y Proyecto de I+D+i Retos MICINN PID2019-108710RB-I00 (2020-2022).

\section{Referencias}

Bernabeu Pellús, A. (2018). "Mi primer BreakOut Edu, una emocionante experiencia: Venciendo a juglareitor”, Comunicación y Pedagogía: nuevas tecnologías y recursos didácticos, núm. 307-308, pp. 36-40.

CABEDO MALlOL, V., CASAR FURIÓ, M. E., GIMÉNEZ CHORNET, V., OLTRA GUTIÉRREZ, J. V. y RAMÓN FERNÁNDEZ, F. (2016). Casos prácticos jurídicos basados en series de animación. Francisca Ramón Fernández (coord.). Valencia: Tirant lo Blanch.

CABEDO MALLOL, V., CASAR FURIÓ, M. E., GIMÉNEZ CHORNET, V., LULl NOGUERA, C., OLTRA GUTIÉRREZ, J. V. y RAMÓN FERNÁNDEZ, F. (2017). Casos prácticos jurídicos basados en series de ficción. Francisca Ramón Fernández (coord.). Valencia: Tirant lo Blanch.

CABEDO MALlOL, V., CASAR FURIÓ, M. E., GIMÉNEZ CHORNET, V., LULl NOGUERA, C., OLTRA GUTIÉRREZ, J. V. y RAMÓN FERNÁNDEZ, F. (2018). Casos prácticos jurídicos basados en documentales. Francisca Ramón Fernández (coord.). Valencia: Tirant lo Blanch. 
El aprendizaje mediante BreakoutEdu en ciencias jurídicas: humor y juego en el aula. Una experiencia en la Universitat Politècnica de València

Cabedo Mallol, V., Casar Furió, M. E., Giménez Chornet, V., Lull Noguera, C., Oltra Gutiérrez, J. V., Osete Cortina, L., RAMÓn fernández, F. y Soriano Soto, Mª. D. (2019). Casos prácticos jurídicos basados en el humor. Francisca Ramón Fernández (coord.). Valencia: Tirant lo Blanch.

CORCHUElo RODRÍGUEZ, C. A. (2018). “Gamificación en educación superior: experiencia innovadora para motivar estudiantes y dinamizar contenidos en el aula”, Edutec. Revista Electrónica de Tecnología Educativa, núm. 63, pp. 29-41.

$<$ https://doi.org/10.21556/edutec.2018.63.927> [Consulta: 05 de marzo de 2020].

García Peralta, A. (2018). “Gamificación, Escape Room o BreakOut Edu?”, Comunicación y Pedagogía: nuevas tecnologías y recursos didácticos, núm. 307-308, pp. 17-20.

Liarte Alcaine, Ma. R. (2018). "BreakOut Edu de la Revolución industrial”, Comunicación y Pedagogía: nuevas tecnologías y recursos didácticos, núm. 309-310, pp. 16-20.

ManZano-León, A., SÁnchez-SÁnchez, M., Trigueros-ramos, R., Álvarez-Hernández, J. y Aguilar-ParRA, J. M. (2020). "Gamificación y Breakout Edu en Formación Profesional. El programa «Grey Place» en Integración Social”, EDMETIC, Revista de Educación Mediática y TIC, vol. 9, núm. 1, pp. 1-20.

<https://doi.org/10.21071/edmetic.v9i1.12067> [Consulta: 05 de marzo de 2020].

Moreno Fuentes, E. (2019). "El «breakoutedu" como herramienta clave para la gamificación en la formación inicial de maestros/as», Edutec. Revista Electrónica de Tecnología Educativa, núm. 67, pp. 66-79.

$<$ https://www.edutec.es/revista/index.php/edutec-e/article/view/1247/665 $>$ [Consulta: 05 de marzo de 2020].

Moreno Fuentes, E., Perales molada, R. Ma. e Hidalgo Navarrete, J. (2019). "Estudio cualitativo sobre el uso de la gamificación en Educación Superior para promover la motivación en el alumnado", Aula de encuentro: Revista de investigación y comunicación de experiencias educativas, vol. 21, núm. 2, pp. 5-26.

$<$ https://revistaselectronicas.ujaen.es/index.php/ADE/article/view/5117/4645> [Consulta: 04 de marzo de 2020].

NEGREE, C. (2017). "BreakoutEdu”, microgamificación y aprendizaje significativo.

$<\underline{\text { https://www.educaweb.com/noticia/2017/07/26/breakoutedu-microgamificacion-aprendizaje- }}$ significativo-15068/ > [Consulta: 21 de julio de 2020].

O’Brien, K., \& Pitera, J. (2019). Gamifying Instruction and Engaging Students With Breakout EDU. Journal of Educational Technology Systems, 48(2), 192-212.

OLTRA GUTIÉRREZ, J. V., RAMÓN FERNÁNDEZ, F., CABEDO MALlOL, V., CASAR FURIÓ, M. E., y GIMÉNEZ CHORNET, v. (2016). "Uso de fragmentos de películas y series como herramientas de innovación docente. Una experiencia con alumnos de informática", en INRED. II Congreso Nacional de Innovación Educativa y Docencia en Red, Universitat Politècnica de València.

PÉREZ-LÓPEZ, I. y Rivera GARCíA, E. (2017). "Formar docentes, formar personas: análisis de los aprendizajes logrados por estudiantes universitarios desde una experiencia de gamificación", Signo y Pensamiento, vol. 36, núm. 70, pp. 112-129.

(cc) EY-NC-ND 2020, Universitat Politècnica de València

Congreso In-Red (2020) 
$<$ https://www.edutec.es/revista/index.php/edutec-e/article/view/1247/665 $>$ [Consulta: 05 de marzo de 2020].

PRIETO ANDREU, J. M. (2020). “Una revisión sistemática sobre gamificación, motivación y aprendizaje en universitarios", Teoría de la educación, vol. 32, núm. 1, 2020, pp. 73-199.

$<$ https://revistas.usal.es/index.php/1130-3743/article/view/teri.20625/21290> [Consulta: 17 de julio de 2020].

Queiruga-Dios, A., Santos SÁnchez, M. J., Queiruga Dios, M., Gayoso Martínez, V., \& Hernández ENCINAS, A. (2020). “A Virus Infected Your Laptop. Let's Play an Escape Game”. Mathematics, 8(2), 166.

RAMÓN FERNÁNDEZ, F., CABEDO MALLOL, V., CASAR FURIÓ, M. E., GIMÉNEZ CHORNET, V., LULL NOGUERA, C. y OLTRA GUTIÉRREZ, J. V. (2016a). "Diseño de casos prácticos jurídicos basados en series de animación. Una innovación docente con soporte audiovisual”, en Derecho y TIC: últimas innovaciones docentes, Coordinadores Ana María Delgado García e Ignacio Beltrán de Heredia Ruiz. Barcelona: Huygens editorial, pp. 123-132.

RAMÓN FERNÁNDEZ, F., CABEDO MALLOL, V., CASAR FURIÓ, M. E., GIMÉNEZ CHORNET, V., y OLTRA GUTIÉRREZ, J. V. (2016b). "Utilización de las series de animación como recurso didáctico en la elaboración de casos. Análisis de una experiencia en la innovación docente en la Universitat Politècnica de València", en INRED. II Congreso Nacional de Innovación Educativa y Docencia en Red, Universitat Politècnica de València.

Ramón Fernández, F., Lull Noguera, C., Soriano Soto, Ma. D., Cabedo Mallol, V., Casar Furió, M. E., Giménez Chornet, V., Oltra Gutiérrez, J. V. y Orduña Malea, E. (2019a). "Diseño de materiales docentes basados en recursos audiovisuales de humor para la mejora del aprendizaje y su evaluación en ciencias sociales y jurídicas. Análisis del diseño de la actividad sobre las casas Cueva y «Los Picapiedra» en La docencia del Derecho en la sociedad digital, Barcelona: Huygens Editorial.

RAmón Fernández, F., Lull Noguera, C., Soriano Soto, Ma. D. (2019b). “Actividades docentes del PYME. Diseño de materiales docentes para la mejora del aprendizaje y su evaluación en ciencias del suelo, sociales y jurídicas" en XXXII Reunión Nacional de Suelos, 10 a 13 de septiembre de 2019 RENS. Sevilla: Sociedad Española de la Ciencia del Suelo.

Ramón Fernández, F., Lull Noguera, C., Soriano Soto, Ma.D., Cabedo Mallol, V., Casar Furió, Mª. E., Giménez Chornet, V., Oltra Gutiérrez, J. V. y Osete Cortina, L. (2020). “Magia y humor en las aulas: experiencias con «Breakoutedu» en la Universitat Politècnica de València" en La innovación del Derecho en línea: cuando la innovación se convierte en necesidad. Barcelona: Huygens Editorial,pp. 381-395.

$<$ http://symposium.uoc.edu/_files/_event/_38228/_editorFiles/file/DERECHO_TIC_2020_NAVEGABL E\%20(1).pdf > [Consulta: 17 de julio de 2020].

Rouse, W. (2017). “Lessons learned while escaping from a zombie: designing a breakout edu game”. The History Teacher, 50(4), 553-564. 